\title{
LEVANTAMENTO PRELIMINAR DAS LARVAS DE PORCELLANIDAE (DECAPODA, ANOMURA) DO SISTEMA ESTUARINO DOS RIOS PIRAQUÊ-AÇÚ E PIRAQUÊ-MIRIM, ARACRUZ, ESPÍRITO SANTO*
}

\author{
MAGRIS, R. A. ${ }^{1} \&$ LOUREIRO FERNANDES, L. ${ }^{2}$ \\ ${ }^{1}$ Bolsista CNPq-Pibic. E-mail: ra_magris@yahoo.com.br \\ ${ }^{2}$ Universidade Federal do Espírito Santo - Depto. de Ecologia e Recursos Naturais - Av. Fernando \\ Ferrari s/n - CEP29060-900 - Vitória, ES.
}

\begin{abstract}
Magris, R.A. \& Loureiro Fernandes, L. 2005. Preliminary survey of Porcellanidae larvae at the Piraquê-açú river system estuary, Aracruz, Espírito Santo State. Braz. J. Aquat. Sci. Technol. 9(1):49-54. ISSN 1808-7035. Composition and abundance of Porcellanidae larvae were studied at the Piraquê-açú and Piraquê-mirim estuarine system, in the city of Aracruz, state of Espírito Santo. These larvae were collected monthly (between April and July 2003) in eight sampling locations, with 4 locations at the Piraquê-açú River and 4 at the Piraquê-mirim River. Samples were collected using a plankton net with $50 \mathrm{~cm}$ mouth opening and 200 micron mesh size. Abiotic factors (e.g. temperature and salinity) were also analyzed in situ at each sampling location and water was sampled with a Van Dorn bottle to analyze chlorophyll-a concentration. Two species (Petrolisthes armatus and Pachycheles monilifer) and one genus (Megalobrachium) were found. Piraquê-açú River showed a greater variation in salinity among the diferent sampling locations and a higher abundance of larvae. The highest larvae abundance was registered in April. P. armatus and Megalobrachium sp. were found at the zoea I and zoea II stages. Significant differences between species (and their developmental stages), sampling locations and rivers were not observed. Diffences were observed only between larvae abundance and months. Spearman's correlation evidenced significant correlation between larvae at the zoea I stage and chlorophyll-a concentration, indicating that early life stages feed mainly on phytoplankton.
\end{abstract}

Keywords: larvae, Porcellanidae, abundance, estuary, Espírito Santo.

\section{INTRODUÇÃO}

A zona costeira concentra uma enorme diversidade de recursos naturais renováveis e a grande maioria da população humana (Kennish, 1990). Sua progressiva ocupação, muitas vezes de forma desordenada e acelerada ao longo das últimas décadas, tem gerado conflitos e problemas relativos aos mais diversos aspectos, tais como à pesca, maricultura, biodiversidade, qualidade ambiental, erosão e uso e ocupação costeira. O conhecimento da diversidade e da estrutura dos ecossistemas costeiros representa, desta forma, caráter fundamental na conservação dos mesmos, gerando informações úteis para gerenciar e restaurar tais sistemas e seus recursos (Omori \& Ikeda, 1992).

Neste aspecto, a estrutura e a distribuição de comunidades planctônicas têm sido cada vez mais estudados não só pela importância ecológica, mas também porque são utilizadas como indicadores de qualidade ambiental (Parsons et al., 1984).

\footnotetext{
*Trabalho financiado pelo Projeto RECOS - Instituto do Milênio MCT/CNPq/PADCTIII
}

Este estudo tem como objetivo realizar um levantamento de informações sistemáticas e ecológicas sobre a ocorrência das larvas de Porcellanidae no estuário dos rios Piraquê-açú e Piraquê-mirim, possibilitando a obtenção de informações ainda sem precedentes na região.

\section{MATERIAL E MÉTODOS}

O estuário dos rios Piraquê-açú e Piraquê-mirim está localizado na região próxima ao distrito de Santa Cruz (4009'W e $19^{\circ} 57^{\prime}$ 'S), município de Aracruz, ES, a $50 \mathrm{~km}$ ao norte da cidade de Vitória. A estrutura deste sistema é bastante peculiar e, próximo à localidade de Santa Cruz, os rios se encontram numa bifurcação em Y unindo seu percurso rumo ao mar. O estuário possui uma área de $6 \mathrm{Km}^{2}$ e um manguezal que ocupa 12.3 $\mathrm{Km}^{2}$. A amplitude de maré do litoral capixaba varia de 1,40 a 1,50 m e o estuário dos rios Piraquê-açú e Piraquê-mirim classifica-se como fracamente estratificado.

O zooplâncton foi coletado mensalmente entre abril e julho de 2003 em oito pontos amostrais, sendo 
quatro ( $\mathrm{Cl}$ a CIV) no braço sul do estuário (rio Piraquêmirim) e quatro (EI a EIV) no braço norte (rio Piraquêaçú) sendo que em ambos, o ponto I foi o mais interno e o ponto IV o mais próximo da desembocadura do estuário (Figura 1).

Arrastos horizontais subsuperficiais foram realizados utilizando-se uma rede de plâncton cônica de 50 $\mathrm{cm}$ de boca e malha de 200 micrômetros. O material coletado foi fixado em solução aquosa de formalina $5 \%$ tamponada com tetraborato de sódio para posterior análise em laboratório. As variáveis abióticas (temperatura e salinidade) foram obtidas in situ. As amostras de água para determinação da concentração de clorofila-a foram coletadas com uma garrafa de Van Dorn. Na análise da clorofila-a, a extração desta foi feita no escuro utilizando o método do etanol $95 \%$ à $80^{\circ} \mathrm{C}$, segundo Nusch \& Palme (1975).

As larvas de Porcellanidae foram tríadas, contadas e identificadas até o menor nível taxonômico possível utilizando literatura pertinente (Boschi, 1981 e 1996; Gore, 1972 e 1973).

Foram determinados os valores de abundância das espécies e dos seus respectivos estágios larvais presentes nas amostras. A normalidade dos dados de abundância foi testada pelo teste de KolmogorovSmirnov-Lilliefors e a abundância das espécies foi eventualmente normalizada com log-transformação. Análises de variância (ANOVAs) do tipo III, foram conduzidas sobre as densidades totais de cada espécie e de cada estágio larval para testar as diferenças entre os meses de coleta, pontos de amostragem e rios. A homogeneidade das variâncias entre os meses e pontos foi verificada através do teste de Duncan e entre os rios com o teste de Bonferroni. Foi realizada a análise da correlação não paramétrica de Spearman para verificar o grau de correlação entre a abundância das espécies e de seus estágios larvais e a salinidade e clorofila-a.

\section{RESULTADOS E DISCUSSÃO}

As variáveis abióticas medidas evidenciaram que o estuário do rio Piraquê-açú apresenta características típicas de estuários tropicais com variações mais evidentes de suas características físico-químicas (maiores variações na temperatura e salinidade) enquanto o estuário do rio Piraquê-mirim apresentou características de águas costeiras, uma vez que seu aporte fluvial é bastante reduzido.

Enquanto a salinidade apresentou pouca variação nos seus valores ao longo dos pontos do estuário, apresentando apenas um pequeno aumento à jusante, a concentração de clorofila-a apresentou os maiores valores nos pontos mais à montante e declinou suas concentrações na porção mais externa do estuário.

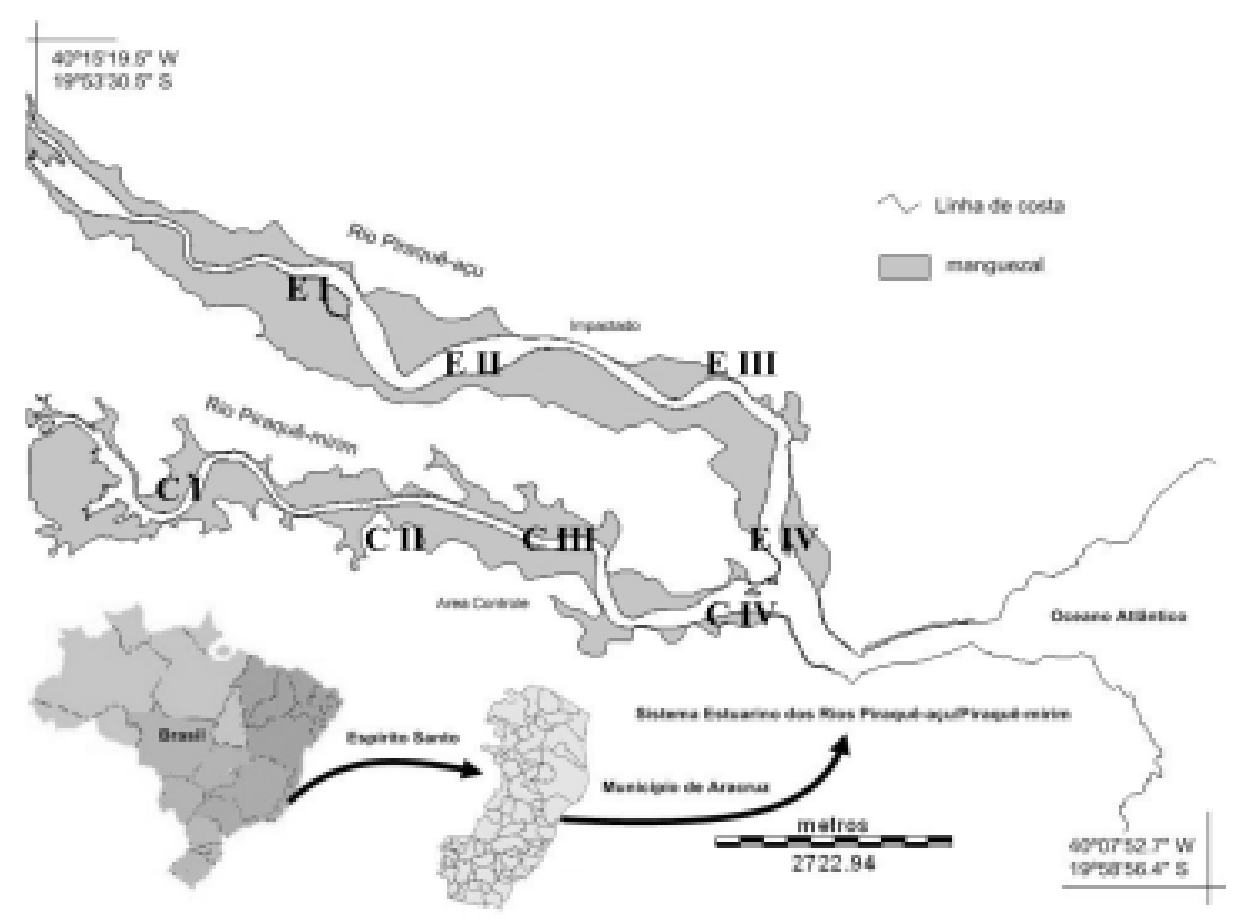

Figura 1 - Mapa da região de estudo incluindo os pontos amostrais E I a E IV (Piraquê-açú) e C I a C IV (Piraquê-mirim). 
(Figura 2). Barroso (2004) também evidenciou o mesmo padrão neste sistema estuarino, atribuindo o aporte de água doce como fonte de nutrientes alóctones e matéria orgânica para o ambiente estuarino.

Foram encontradas 2 espécies (Petrolisthes armatus e Pachycheles monilifer) e 1 gênero (Megalobrachium) da família Porcellanidae, todos de habitats costeiros. $P$. armatus foi a espécie mais abundante e com maior constância na sua ocorrência, chegando a representar até mesmo $100 \%$ dos organismos capturados em alguns pontos. Isto poderia indicar que o período reprodutivo da espécie compreendeu todo período amostral, conforme demonstrado por Epifanio \& Dittel (1984) em um estuário tropical na Costa Rica.

Enquanto $P$. armatus diminui a abundância gradativamente entre os meses de coleta com pico de abundância no mês de abril, Megalobrachium sp. apresentou maior abundância em maio e só ocorreu nos pontos mais à montante (Figura 3). P. monilifer, por sua vez, foi a espécie menos abundante e somente encontrada nos pontos mais próximos à desembocadura.

$P$. armatus foi encontrada nos estágios de desenvolvimento de zoea 1 e zoea 2 (Figura 4). Tanto o estágio de zoea 1 quanto o estágio de zoea 2 declinaram suas densidades ao longo dos meses de coleta, permanecendo aproximadamente a mesma proporção entre ambos. Foram registrados também os mesmos dois estágios de desenvolvimento para Megalobrachium $s p$. e apenas o estágio de zoea 2 para $P$. monilifer.

Muitos autores (Lambert \& Epifanio, 1982; Brookins \& Epifanio, 1985) têm associado a presença de larvas nos primeiros estágios de desenvolvimento nas camadas subsuperficiais da coluna d'água com o processo de exportação de biomassa para as águas costeiras. Esta estratégia indica que as larvas não completam seu ciclo de vida larval no estuário, migrando para regiões costeiras próximas.

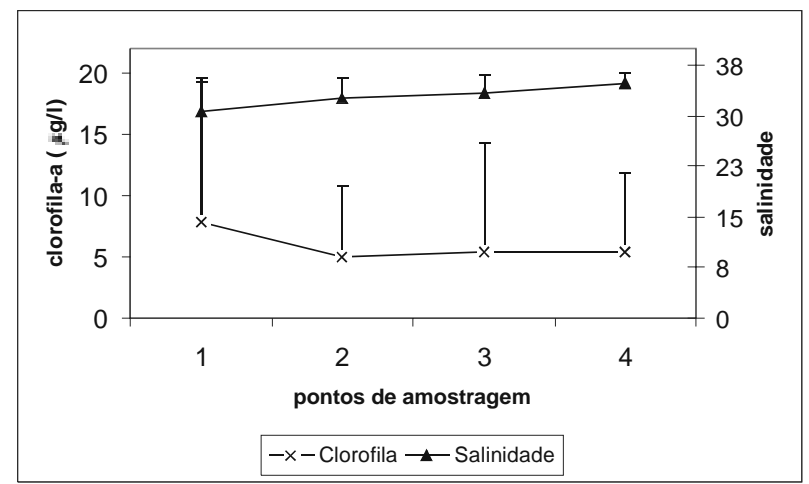

Figura 2 - Valores médios da concentração de clorofila-a e da salinidade ao longo dos pontos de amostragem no estuário dos rios Piraquê-açú e Piraquê-mirim. 1 - ponto mais interno; 4 - ponto mais externo. As barras de erro indicam o desvio padrão.

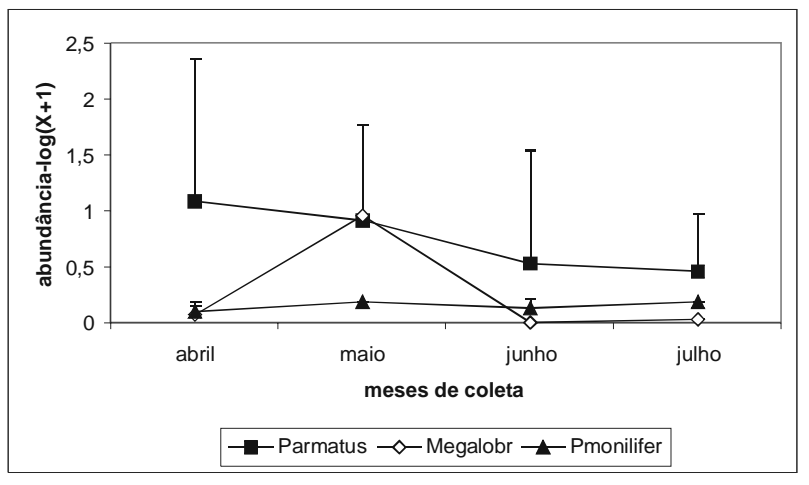

Figura 3 - Variações da abundância log $(x+1)$ dos taxa de Porcellanidae nos meses de coleta do estuário dos rios Piraquêaçú e Piraquê-mirim. Parmatus - Petrolisthes armatus; Megalobr Megalobrachium sp.; Pmonilifer - Pachycheles monilifer. As barras de erro indicam o desvio padrão.

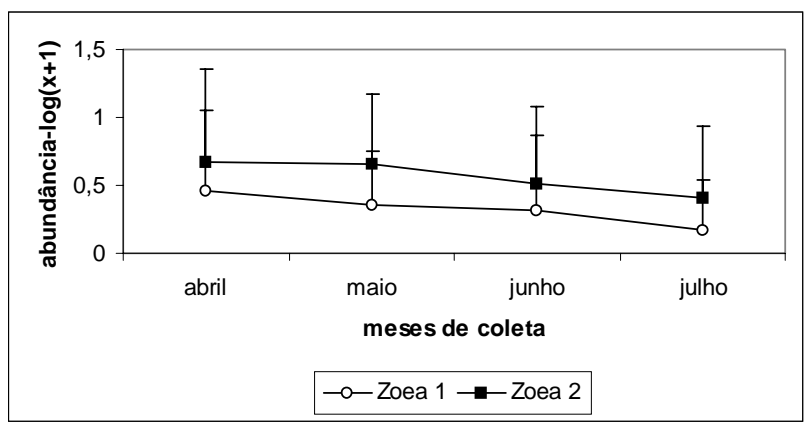

Figura 4 - Abundância média log $(x+1)$ dos dois estágios de desenvolvimento (zoea 1 e zoea 2 ) da principal espécie de Porcellanidae (Petrolisthes armatus) ao longo dos meses de coleta do estuário dos rios Piraquê-açú e Piraquê-mirim. As barras de erro indicam o desvio padrão.

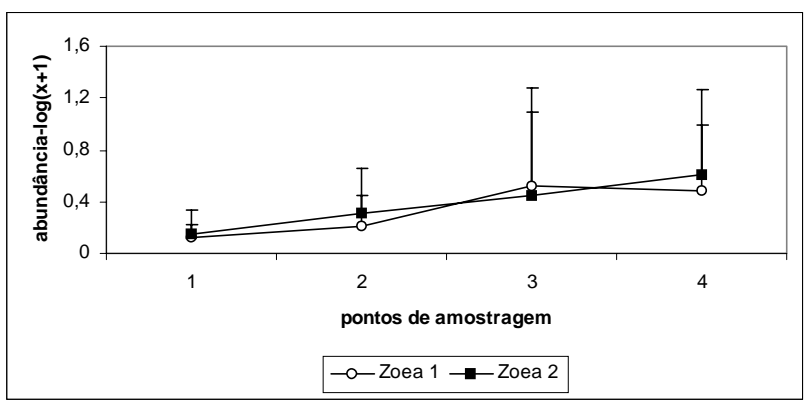

Figura 5 - Abundância média log $(x+1)$ dos dois estágios de desenvolvimento (zoea 1 e zoea 2 ) das larvas de Porcellanidae ao longo dos pontos de amostragem no estuário dos rios Piraquê-açú e Piraquê-mirim. 1 - ponto mais interno; 4 - ponto mais externo. As barras de erro indicam o desvio padrão. 
Magris \& Loureiro Fernandes: Levantamento preliminar das larvas de Porcellanidae em um sistema estuarino tropical.

Tabela 1 - ANOVA sobre a abundância (ind. $\mathrm{m}^{-3}$ ) das espécies e estágios larvais de Porcellanidae ao longo dos meses, rios e pontos do estuário dos rios Piraquê-açú e Piraquê-mirim. Ns: não significante com a = 0,05.

\begin{tabular}{|c|c|c|c|c|c|c|}
\hline \multirow{2}{*}{$\begin{array}{c}\text { Taxa } \\
\text { (variáveis } \\
\text { dependentes) }\end{array}$} & \multicolumn{3}{|c|}{ Variáveis independentes } & \multicolumn{3}{|c|}{ Grupos homogêneos } \\
\hline & Mês & Rio & Ponto & Mês (Duncan) & $\begin{array}{c}\text { Rio } \\
\text { (Bonferroni) }\end{array}$ & $\begin{array}{c}\text { Ponto } \\
\text { (Duncan) }\end{array}$ \\
\hline $\begin{array}{c}\text { Petrolisthes } \\
\text { armatus (zoea 1) }\end{array}$ & Ns & Ns & Ns & - & - & - \\
\hline $\begin{array}{c}\text { Petrolisthes } \\
\text { armatus (zoea 2) }\end{array}$ & 0,029 & Ns & Ns & $\begin{array}{l}\text { Abr, mai }>\text { jul, } \\
\text { jun }\end{array}$ & - & - \\
\hline $\begin{array}{c}\text { Petrolisthes } \\
\text { armatus (total) }\end{array}$ & 0,035 & Ns & Ns & $\begin{array}{l}\text { Abr, mai > } \\
\text { mai, jul, jun }\end{array}$ & - & - \\
\hline $\begin{array}{l}\text { Megalobrachium } \\
\text { sp. (zoea 1) }\end{array}$ & Ns & Ns & Ns & - & - & - \\
\hline $\begin{array}{l}\text { Megalobrachium } \\
\text { sp. (zoea 2) }\end{array}$ & Ns & Ns & Ns & - & - & - \\
\hline $\begin{array}{l}\text { Megalobrachium } \\
\text { sp. (total) }\end{array}$ & Ns & Ns & Ns & - & - & - \\
\hline $\begin{array}{c}\text { Pachycheles } \\
\text { monilifer (zoea 2) }\end{array}$ & Ns & Ns & Ns & - & - & - \\
\hline $\begin{array}{l}\text { Total de larvas } \\
\text { (zoea 1) }\end{array}$ & 0,044 & Ns & Ns & $\begin{array}{l}\text { Abr, mai > } \\
\text { mai, jun, jul }\end{array}$ & - & - \\
\hline $\begin{array}{c}\text { Total de larvas } \\
\text { (zoea 2) }\end{array}$ & 0,015 & Ns & Ns & $\begin{array}{l}\text { Abr, mai > } \\
\text { mai, jul, jun }\end{array}$ & - & - \\
\hline $\begin{array}{c}\text { Abundância total } \\
\text { de larvas }\end{array}$ & 0,021 & Ns & Ns & $\begin{array}{l}\text { Abr, mai > } \\
\text { mai, jul, jun }\end{array}$ & - & - \\
\hline
\end{tabular}

A ANOVA sobre a abundância das espécies e dos seus estágios larvais bem como da abundância total de larvas e estágios de desenvolvimento ao longo dos meses, rios e pontos somente apresentou diferenças significativas entre os meses $(p<0,05)$ (Tabela 1$)$. Não foi possível então estabelecer um padrão de distribuição espacial destas larvas. Para $P$. armatus e para a abundância total de Porcellanidae em ambos estágios de desenvolvimento, a maior abundância esteve associado ao mês de abril, sendo relacionado ao pico de reprodução destes organismos.

Embora a proporção média de larvas no estágio de zoea 1 diminuiu gradativamente entre os pontos à medida que se aproximou da região de desembocadura do estuário, a abundância total destes dois estágios aumentou consideravelmente à jusante (Figura 5).

Através da correlação não-paramétrica de Spearman entre a abundância das espécies e de seus estágios larvais bem como da abundância total de larvas e dos estágios de desenvolvimento revelou que não houve nenhuma correlação significativa entre a abundância destes organismos com a salinidade (Tabela 2). No entanto, houve correlação significativa a nível de 0,05 entre $P$. armatus no estágio de zoea 1 com a concentração de clorofila-a, bem como entre a abundância total de larvas no estágio de zoea 1 e a clorofila-a. E ainda houve correlação significativa a nível de 0,01 entre Megalobrachium sp. no estágio de zoea 1 e a concentração de clorofila-a. Por outro lado, não houve cor- relação significativa (a 0,01 ou a 0,05$)$ entre a concentração de clorofila-a e nenhuma larva no estágio de zoea 2. Isto pode sugerir que o fitoplâncton funciona como componente importante da dieta das larvas de Porcellanidae no primeiro estágio de seu desenvolvimento, mas não desempenha papel principal na alimentação dos Porcellanidae nos estágios avançados de desenvolvimento. Seeliger et al. (1998) já documentaram a marcada variação ontogenética na dieta das larvas de alguns decápodos. De acordo com GonzálezGordillo e Rodríguez (2003), as larvas de Decapoda são capazes de se alimentar de pequenas espécies de fitoplâncton, porém, a maior disponibilidade de pequenos herbívoros, como os copépodos, após a alta produção de fitoplâncton, pode ser mais eficaz ao seu desenvolvimento.

Perissinoto et al. (2000) também observaram padrões de distribuição de alguns componentes do zooplâncton ligados à disponibilidade de fitoplâncton, influenciando diretamente na abundância dos organismos, no crescimento e no clico de vida destes no ambiente.

Desta maneira, alguns autores (Fernandes et al., 2002; Neumann-Leitão et al., 1999) destacam a importância dos estudos de acompanhamento da biomassa e composição destas larvas para compreensão das relações tróficas nestes ambientes e das suas mudanças em resposta às condições de eutrofização artificial que muitos estuários estão propensos. 
Braz. J. Aquat. Sci. Technol., 2005, 9(1):49-54.

Tabela 2 - Correlação não paramétrica de Spearman entre es espécies e seus estágios larvais e a salinidade e a concentração de clorofila-a do estuário dos rios Piraquê-açú e Piraquê-mirim.

\begin{tabular}{|c|c|c|}
\hline \multirow[t]{2}{*}{ Taxas } & \multicolumn{2}{|c|}{ Correlação não-paramétrica de Spearman } \\
\hline & Salinidade & Clorofila-a \\
\hline $\begin{array}{l}\text { Petrolisthes armatus } \\
\text { (zoea 1) }\end{array}$ & $-0,239$ & $0,634^{\star \star}$ \\
\hline $\begin{array}{c}\text { Petrolisthes armatus } \\
\text { (zoea 2) }\end{array}$ & $-0,268$ & 0,282 \\
\hline $\begin{array}{c}\begin{array}{c}\text { Petrolisthes armatus } \\
\text { (total) }\end{array} \\
\end{array}$ & $-0,312$ & $0,393^{*}$ \\
\hline $\begin{array}{c}\text { Megalobrachium sp. } \\
\text { (zoea 1) }\end{array}$ & $-0,263$ & $0,378^{\star}$ \\
\hline $\begin{array}{c}\text { Megalobrachium sp. } \\
\text { (zoea 2) }\end{array}$ & $-0,101$ & 0,195 \\
\hline $\begin{array}{c}\text { Megalobrachium sp. } \\
\text { (total) }\end{array}$ & $-0,106$ & $0,380^{*}$ \\
\hline $\begin{array}{c}\text { Pachycheles monilifer } \\
\text { (zoea 2) }\end{array}$ & 0,180 & $0,364^{*}$ \\
\hline $\begin{array}{c}\text { Total de larvas } \\
\text { (zoea 1) }\end{array}$ & $-0,239$ & $0,634^{\star \star}$ \\
\hline $\begin{array}{l}\text { Total de larvas } \\
\text { (zoea 2) }\end{array}$ & $-0,259$ & 0,296 \\
\hline $\begin{array}{c}\text { Abundância total de } \\
\text { larvas }\end{array}$ & $-0,321$ & $0,408^{*}$ \\
\hline
\end{tabular}

\section{CONCLUSÃO}

A salinidade apresentou pouca variação nos seus valores ao longo dos pontos do estuário, apresentando apenas um pequeno aumento à jusante, e a concentração de clorofila-a apresentou os maiores valores nos pontos mais à montante. Foram encontradas 2 espécies (Petrolisthes armatus e Pachycheles monilifer) e 1 gênero (Megalobrachium) da família Porcellanidae, todos de habitats costeiros. $P$. armatus foi a espécie mais abundante e com maior constância na sua ocorrência. Todos os taxa foram encontrados nos primeiros estágios de desenvolvimento (zoea 1 e zoea 2). A proporção média de larvas no estágio de zoea 1 diminuiu gradativamente entre os pontos à medida que se aproximou da região de desembocadura do estuário, indicando que os estágios larvais iniciais se alimentam preferencialmente de fitoplâncton.

\section{AGRADECIMENTOS}

Ao Projeto RECOS Instituto do Milênio - MCT/ CNPq/PADCTIII pelo financiamento dos trabalhos e da bolsa de Iniciação Científica. Ao Departamento de Ecologia e Recursos Naturais pela infra-estrutura e aos colegas do Projeto pelo apoio durante as coletas de campo.

\section{REFERÊNCIAS}

Barroso, G.F. 2004. Development of an evaluation framework for sustainable bivalve aquaculture: a strategic plan approach in Espírito Santo, Brazil. Tese de Doutorado. University of Victoria. $128 \mathrm{p}$.

Boschi, E.E. 1981. Larvas de Crustacea Decapoda. In: D. Boltovskoy (ed.) Atlas del Zooplancton del Atlantico Sudoccidental. INIDEP. 699-758pp.

Boschi, E.E. 1996. Larvas de Crustacea Decapoda. In: R. Gasca \& E.S. Morales (eds.) Introducion al estudio del zooplancton marino. El Colegio de la Frontera Sur (ECOSUR)/CONACYT. 343-374pp.

Brookins, K.G. \& Epifanio, C.E. 1985. Abundance of Brachyura Larvae in a Small Coastal Inlet over Six Consecutive Tidal Cycles. Estuaries. 8(1):60-67.

Epifanio, C.E. \& Dittel, A.I. 1984. Seasonal Abundance of Brachyuran Crab Larvae in a Tropical Estuary: Gulf of Nicoya, Costa Rica, Central América. Estuaries. 7(4B):501-505.

Fernandes, L.D.; Bonecker, S.L.C. \& Valentin, J.L. 2002. A Dynamic of Decapod Crustacean Larvae on the entrance of Guanabara Bay. Brazilian Archives of Biology and Technology. 45(4):491-498.

González-Gordillo, J.I. \& Rodríguez, A. 2003. Comparative seasonal and spatial distribution of decapod larvae assemblages in three coastal zones 
of the south-western Iberian Peninsula. Acta Oecologica. 24:S219-S233.

Gore, R.H. 1972. Petrolisthes armatus (Gibbes, 1850): The development under laboratory conditions of larvae from a Pacific specimen (Decapoda, Porcellanidae). Crustaceana. 22(1):67-83.

Gore, R.H. 1973. Pachycheles monilifer (Dana, 1852): The development in the laboratory of larvae from na Atlantic specimen with a discussion of some larval characters in the genus (Crustacea: Decapoda; Anomura). Biology Bulletin. 144:132-150.

Kennish, M.J. 1990. Ecology of Estuaries: Anthropogenic Effects. CRC Press, Boca Raton, 494p.

Lambert, R. \& Epifanio, C.E.A. 1982. Comparison of Dispersal Strategies in Two Genera of Brachyuran crab in a Secondary Estuary. Estuaries. 3:182-188.

Neumann-Leitão, S.; Paranaguá, M.N.; NascimentoVieira, D.A.; Koening, M.L. \& Gusmão, L.M. 1999. Plankton disturbance at Suape estuarine area (Pernambuco, Brazil) after a complex port implantation. Ecological Sustainable Development. 2:46-56.
Nusch, E.A. \& Palme, G. 1975. Biologische methoden fur die praxis der gewisseruntersuchung 1. Bestimmung des Chlorophyll a und Phaeopigmentgehaltes in Oberflachenwasser. GWF. 116(12):562-565.

Omori, M. \& Ikeda, T. 1992. Methods in Marine Zooplankton Ecology. Krieger Plubishing Company, Florida, 329p.

Parsons, T.R.; Takahashi, M. \& Hargrave, B. 1984. Biological Oceanographic Processes. $3^{\circ}$ Edição. Pergamon Press, Oxford, 330p.

Perissinotto, R.; Walker, D.R.; Webb, P.; Wooldridge, T.H. \& Bally, R. 2000. Relationships between Zooand Phytoplankton in a warm-temperate, semipermanently closed estuary, South Africa. Estuarine, Coastal and Shelf Science. 51:1-11.

Seeliger, U.; Costa, C.S.B. \& Abreu, P.C. 1998. Fluxo de Energia e Habitats no estuário da Lagoa dos Patos, p. 199-203. In: Seeliger, U; Costa, C.S.B. \& Abreu, P.C. (orgs.) Os Ecossistemas Costeiro e Marinho do Extremo Sul do Brasil. Ecoscientia. 199203pp. 